\title{
Erratum to: Postural adjustment errors during lateral step initiation in older and younger adults
}

\author{
Patrick J. Sparto • Susan I. Fuhrman • Mark S. Redfern • \\ Subashan Perera $\cdot$ J. Richard Jennings $\cdot$ \\ Alia A. Alghwiri · Joseph M. Furman
}

Published online: 18 January 2015

(c) Springer-Verlag Berlin Heidelberg 2015

\section{Erratum to: Exp Brain Res (2014) 232:3977-3989 DOI 10.1007/s00221-014-4081-z}

Alia A. Alghwiri was mistakenly omitted as a co-author in the original publication.

The complete author group is given below:

Patrick J. Sparto - Susan I. Fuhrman - Mark S. Redfern · Subashan Perera - J. Richard Jennings - Alia A. Alghwiri . Joseph M. Furman

Additionally, a part of the grant detail was omitted in the acknowledgement section of the original publication. The correct last line should read as:
The online version of the original article can be found under doi:10.1007/s00221-014-4081-z.

\section{P. J. Sparto $(\bowtie) \cdot$ M. S. Redfern · J. M. Furman} Department of Physical Therapy, University of Pittsburgh, 6035, Forbes Tower, Pittsburgh, PA 15260, USA

e-mail:psparto@pitt.edu

P. J. Sparto · S. I. Fuhrman · M. S. Redfern · J. M. Furman Department of Otolaryngology, University of Pittsburgh, Pittsburgh, PA 15260, USA

P. J. Sparto · M. S. Redfern - J. M. Furman Department of Bioengineering, University of Pittsburgh, Pittsburgh, PA 15260, USA

S. Perera

Department of Medicine, University of Pittsburgh, Pittsburgh, PA 15260, USA
This research was supported by funding from the National Institutes of Health (R01 AG031118, P30 AG024827, P30 DC005205), including the Pittsburgh Claude D. Pepper Older Americans Independence Center (P30 AG024827), the Eye and Ear Foundation and the Pennsylvania Lions Hearing Research Foundation, Grant GA-2702 (Principal Investigator: Patrick Sparto).
J. R. Jennings

Department of Psychiatry, University of Pittsburgh, Pittsburgh, PA 15260, USA

J. R. Jennings

Department of Psychology, University of Pittsburgh, Pittsburgh, PA 15260, USA

A. A. Alghwiri

The University of Jordan, Amman, Jordan 